Carlos A. Bordini

Hilton Mariano da Silva

Roberta P. Garbelini

Simone O. Teixeira

José G. Speciali
Received: 2 April 2005

Accepted in revised form: 31 May 2005

Published online: 1 August 2005
C.A. Bordini $\cdot$ H. Mariano da Silva

R.P. Garbelini • S.O. Teixeira

J.G. Speciali (四)

Department of Neurology,

São Paulo University,

School of Medicine at Ribeirão Preto,

Av. Bandeirantes 3900 ,

14049-900 Ribeirão Preto, SP, Brazil

e-mail: hiltonmariano@uol.com.br

Tel.: +55-16-6022548

Fax: +55-16-6330866

\section{Effect of preventive treatment on health-related quality of life in episodic migraine}

\begin{abstract}
The purpose of our study was to prospectively evaluate the impact of preventive drug treatment on the quality of life of patients with episodic migraine (EM). Quality of life evaluations can enhance traditional measures of therapeutic efficacy. Thirty-five consecutive EM without aura patients attending a tertiary care unit (Batatais Headache Clinic) entered the study. They were given a prescription for preventive medication and completed the validated Portuguese version of the generic instrument Short Form-36 (SF-36) questionnaire. Six months later, patients were given another SF-36. We compared the pre-treatment and post-treatment scores for the SF-36 and analysed the headache diaries. The mean age of the 35 patients (32 women and 3 men) was 40.2 years
\end{abstract}

(range 18-60 years). All 35 patients completed the pretreatment and follow-up SF-36. The pre-treatment and post-treatment mean frequency of migraine attacks was 9.16 and 2.4 crises per month, respectively $(p<0.05)$. After the six months of preventive treatment, six of the eight domains evaluated by the SF36 (role physical, bodily pain, general health, vitality, social function and mental health) showed statistically significant $(p<0.05)$ improvement. The preventive treatment of migraine leads to a significant improvement in the quality of life of patients with EM. This improvement was measurable by the general quality of life instrument SF-36.

Key words Headache • Migraine • Health-related quality of life • Preventive treatment $\cdot \mathrm{SF}-36$

\section{Introduction}

Migraine is a common disease that causes a great social and economic burden [1]. There is evidence in the literature that migraineurs fare worse on healt-related quality of life (HRQoL) than patients with other chronic diseases, such as diabetes and hypertension [2] and that migraineurs experience poorer quality of life even between attacks [3,
4]. The Global Burden of Disease Study [5] ranked severe migraine in the highest disability class. The main goal of migraine treatment has evolved from pain relief towards a more comprehensive approach including preventing any migraine symptoms as well as disability. Accordingly, measuring only pain-related aspects such as number of days with certain levels of pain, even though important, may not suffice to understand some other elusive aspects of migraine headache, some of them concerning the 
attacks and others interictal symptoms. Measurements using health-related quality of life (HRQoL) instruments may provide some new insight into the issue. HRQoL represents the global effect of illness and its treatment on a patient, as reported and judged by the patient [6]. This is what distinguishes them from measures of disability [7]. Measurements of HRQoL offer a quantitative assessment of an individual's health status including the aggregate burden imposed by specific health conditions such as migraine headache. HRQoL has been assessed using two categories of questionnaires: generic and disease specific. Generic health status measures are those that purport to be broadly applicable across types and severities of disease, across different medical treatments or health interventions, and across demographic and cultural subgroups. Disease-specific measures are designed to assess specific diagnostic or patient populations, often with the goal of measuring responsiveness or 'clinically' important changes [8]. Accordingly, HRQoL tools have been increasingly accepted as an instrument for assessing therapeutic efficacy and clinical outcome [9].

The pharmacological treatment of migraine may be acute (abortive) and/or preventive (prophylactic). The acute treatment should be able to effect fast and complete relief of pain and the associated symptoms of migraine. The goal of preventive therapy is to reduce the frequency, duration and severity of migraine attacks. The choice of preventive treatment depends on the individual drug's efficacy and side effects, the patient's wants, needs and response to prior treatment, and the presence of any comorbid or coexistent disease. As for acute drugs and their effects on HRQoL, many instruments have been successfully applied, most of them developed by the pharmaceutical companies $[10,11]$. On the other hand, papers concerning preventive migraine therapy and HRQoL tests are rarely seen in the literature. Diamond et al. [12] studied the impact of topiramate (TPM) treatment on HRQoL using two subscales (role-physical and vitality) from the Medical Outcomes Study Short-Form 36 and the Migraine-Specific Quality-of-Life questionnaire (MSQ). The authors enrolled 468 patients, allocated to 4 treatment groups (placebo, TPM 50 mg/day, TPM $100 \mathrm{mg} /$ day, TPM $200 \mathrm{mg} /$ day), and made up the intent-to-treat population. The MSQ Role-Restrictive and Role-Preventive subscales were significantly improved at all TPM doses relative to placebo, and a significant improvement was also shown in the MSQ Emotional Function subscale at 100 or 200 mg/day TPM. The Short Form-36 Role-Physical subscale was significantly improved in both the 100 and 200 $\mathrm{mg}$ /day groups. D' Amico et al. [13] evaluated the effect of preventive treatment for migraine without aura using the Italian versions of the Migraine Disability Assessment Score (MIDAS) questionnaire and the SF-36 survey. After a 2-month run-in period, they were given a prophylactic treatment and re-evaluated after 3 months. Seventy-three patients completed the study. The results showed significant changes after treatment intervention for headache frequency, number of symptomatic drugs and MIDAS score and six scales of SF-36 (except physical function and general health). In order to add new information to this issue, we performed a study as follows.

\section{Subjects and methods}

Thirty-five consecutive episodic migraine (EM) without aura (according to the IHS 1988 criteria) patients attending a tertiary care unit (Batatais Headache Clinic) entered the study. Inclusion criteria were: no previous preventive treatment, ages ranging from 18 to 65 years, history of migraine for at least one year, days with headache frequency from 2 to 14 per month, and, in female patients, the use of an effective contraceptive method was required. Patients with transformed migraine or who were previously diagnosed with chronic diseases known to have influence on HRQoL tool scores such as depression, diabetes, osteoarthritis, congestive heart failure, hypertension, angina, myocardial infarction, asthma or chronic low back pain were excluded. During the initial patient interview, a complete neurological exam was performed and the Portuguese validated version of the generic instrument SF-36 questionnaire was completed [14]. Except for the introduction of the prophylactic treatment, no other changes were made in the current migraine abortive treatment. After the first visit, patients underwent another evaluation 40 days later, and a third on day 90 . In order to assure compliance, we performed a telephone interview once a week. Six months later (last evaluation), patients were again given the SF-36. Pre-treatment and post-treatment variables were compared.

Statistical methods

In order to ascertain if a given variable had a normal distribution, the Kolmorov-Smirnoff test was used. For comparisons between pre- and post-treatment, data with normal distribution were analysed with the $t$-test. The Wilcoxon test was used as a non-parametric alternative.

\section{Results}

The mean age of the 35 patients ( 32 women and 3 men) was 40.2 years $(18-60)$. Fifteen patients were treated with the combination of beta-adrenergic blockers and tricyclic antidepressants (amitriptyline or nortriptyline), five patients received the combination of flunarizine and beta- 


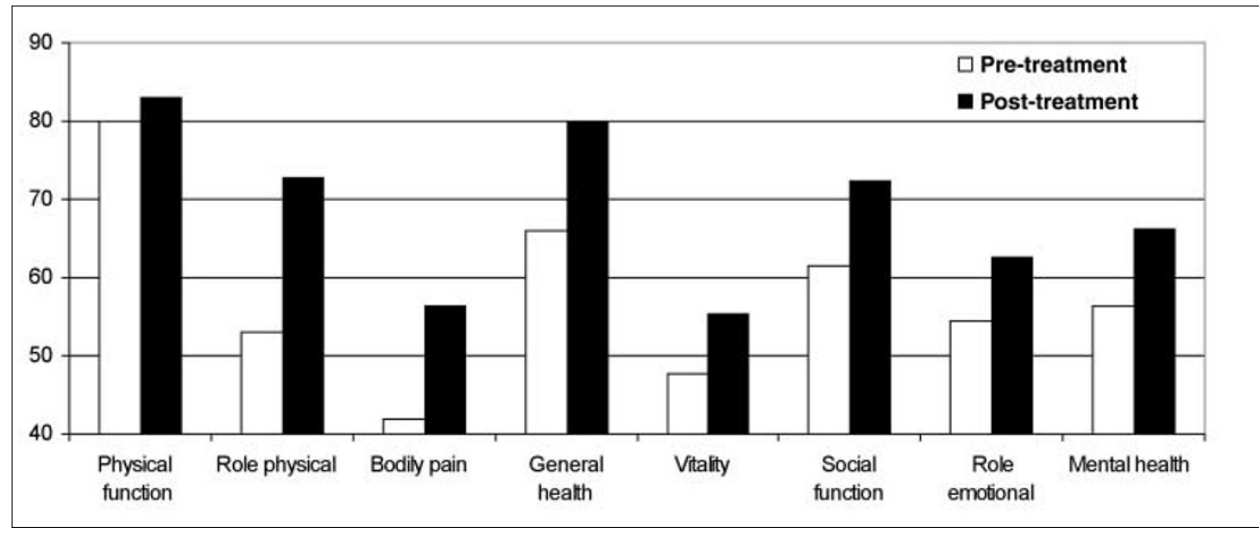

Fig. 1 Comparison of 36 health scores in migraine patients before and after preventive treatment
Table 1 Comparison of the health scales before and after preventive treatment. Data are mean (SD)

\begin{tabular}{lrr}
\hline SF-36 domains & Migraine & $\begin{array}{r}\text { After preventive } \\
\text { treatment }\end{array}$ \\
\hline Physical function & $80(19.8)$ & $83(15.6)$ \\
Role physical* & $53.03(41.8)$ & $72.73(38.7)$ \\
Bodily pain* & $42(26.5)$ & $56.33(23.7)$ \\
General health* & $65.94(22.4)$ & $79.94(20.2)$ \\
Vitality* & $47.58(19.3)$ & $55.3(23.2)$ \\
Social function* & $61.36(26.8)$ & $72.35(22.9)$ \\
Role emotional & $54.44(44.7)$ & $62.63(40.6)$ \\
Mental health* & $56.36(22.1)$ & $66.18(25.3)$ \\
\hline
\end{tabular}

*The variables of SF-36 with statistically significant $(p<0.05)$ differences

adrenergic blockers, three patients were given neuroleptics, three patients were given flunarizine, two patients received the combination of tricyclic antidepressants plus verapamil, two patients were treated with tricyclic antidepressants plus neuroleptics, two patients were given verapamil and two received valproic acid. One patient was treated with a combination of valproic acid and a neuroleptic. There was no modification of prescription due to side effects. The pre-treatment and post-treatment mean frequency of migraine attacks was 9.16 (SD: 3.45, range 3-14) and 2.4 (SD: 2.5, range 0-14) per month, respectively $(p<0.05)$. Six of eight domains evaluated by SF-36 (role physical, bodily pain, general health, vitality, social function and mental health) showed statistically significant $(p<0.05)$ improvement (Table 1 and Fig. 1$)$.

\section{Discussion}

Preventive medications are usually taken daily, whether or not a headache attack is occurring, to reduce the frequency, duration or severity of attacks. The United States
Evidenced Based Guidelines for Migraine [15] has suggested that circumstances that might warrant preventive treatment include: (i) recurring migraine that significantly interferes with the patient's daily routine despite acute treatment (e.g., two or more attacks a month that produce disability that last for 3 days or headache attacks that are infrequent but produce profound disability); (ii) failure of, contraindication to, or troublesome side effects from acute medication; (iii) overuse of acute medication; (iv) special circumstances, such as hemiplegic migraine or attacks with a risk of permanent neurological injury; (v) very frequent headaches (more than two per week), or a pattern of increasing attacks over time, with the risk of developing rebound headache from acute attack medicine; or (vi) patient preference, i.e., the desire to have as few acute attacks as possible. The patients' performance on HRQoL tools has not yet been used to help the indication of preventive treatment.

Choosing the correct preventive drug or combination is a complex task. In order to be more objective in the process, Adelman et al. [16] proposed an analysis that could be used to determine the cost-effectiveness of migraine-preventive medications relative to selected acute-care medications (called "cost-equivalent number"). However, multiple factors can influence this decision, such as patient's age, gender, body weight, side effects of the drug, and comorbid and coexisting diseases. This matter should be regarded as a joint venture between the doctor and the patient, the person who is the real participant in this "single-case scientific experiment" [17, 18]. Drug combinations are commonly used for patients with refractory headache disorders [19] or for patients suffering from some comorbid illness. Nevertheless, our point of view is this approach can be used more frequently and is very useful as the first step of a therapeutic plan in order to obtain a more complete and fast response, such as we performed in this research. Accordingly, some authors have suggested that a combination of drugs with distinct pathophysiological mechanisms could enhance their efficacy [20]. 
How can we evaluate the efficacy of preventive migraine treatment?

The recommendation of the IHS [21] states that evaluation of efficacy should be based on the use of a headache diary and the frequency of attacks per 4 weeks should be the main parameter. Our sample experienced a significant reduction of the frequency of attacks ( 9.16 to 2.4 in a sixmonth follow-up, $p<0.05)$. Despite this recommendation, instruments for measuring HRQoL have been used by some authors in order to incorporate additional information. Dahlöf performed an open, prospective study in order to evaluate the efficacy of abortive treatment with sumatriptan on migraine patient's socio-economic situation, subjective symptoms and general well-being [22]. Ninety-nine patients were included and followed for six months and a significant improvement was observed for the dimensions emotional distress, gastrointestinal symptoms and dizziness, whereas no significant change in the patient's general well-being between attacks, as evaluated by the Minor Symptoms Evaluation Profile instrument, could be demonstrated.

One of the HRQoL's tools is the SF-36. The SF-36 is the most widely used generic instrument for measuring HRQoL. The instrument was developed by the Rand Corporation to assess HRQoL in the Medical Outcomes Study [23, 24] and was translated into numerous languages, and the validity of the eight domains is confirmed in general populations and in a wide variety of patient groups in more than two thousands articles. Solomon et al. [25] studied 147 migraine patients with abortive treatment with sumatriptan and evaluated the effect of the treatment on the HRQoL assessed by the SF-36. After six to nine months of follow-up, the comparison of the pretreatment and the follow-up SF-36 showed significant improvement of three domains of the questionnaire: bodily pain, social function and role functioning. Mushet et al. [26] reported significant improvement of the score of the role emotional dimension of the Short Form-36 after sumatriptan compared to usual therapy. The results of this study showed that the majority of the domains of SF-36 (except for physical function and role emotional) improve with the intervention. The results of this new study are perfectly compatible with the previous observations of D'Amico et al. [13].

This open, longitudinal study aimed to evaluate if a tailored preventive treatment, in a broad sense, could modify some parameters of the quality of life of migraine patients. We did not intend to make head-to-head drug comparisons or to evaluate different drug dosages. The trial was therefore not blinded or randomised, but rather set up to mimic a clinical situation in which the patients are found eligible for treatment with different drugs. Because of the absence of a parallel placebo control group, we could not completely exclude the interference of some variables, such as the patient being seen at a tertiary care centre [27]. It is likely, for example, that the greater attention paid to the patients and the better understanding of the disease by expert physicians, when compared with their previous experiences, have been perceived positively by the patients and may affect treatment results. There may be spontaneous headache remissions or regression towards the mean on HRQoL measurements, especially if patients choose to seek care for headache when they are at their worst. We did not observe dropouts, missed visits or withdrawals. The assessments were made in a selected group of migraine patients recruited from a special Headache Clinic, and so extrapolation of the results to the whole migraine population should be treated with caution.

The preventive medication treatment was used in order to obtain the maximal clinical improvement, as we do in clinical practice. The combination of drugs was used as the first option in the majority of patients. Despite those shortcomings, an important improvement in the quality of life was found in our group of patients, giving support to the current view that tailored treatment is a useful tool for migraine management. Further studies are necessary to confirm our findings.

Acknowledgement The authors are very grateful to Professor Alan M. Rapoport, Columbia University College of Physicians \& Surgeons, New York, US, for a critical reading of the manuscript.

\section{References}

1. Lipton RB, Hamelsky SW, Stewart WF (2001) Epidemiology and impact of headache. In: Silberstein SD, Lipton RB, Dalessio DJ (eds) Wolff's headache and other head pain. Oxford University Press, New York, pp 85-107
2. Osterhaus JT, Townsend RJ, Gandek B, Ware JE Jr (1994) Measuring the functional status and well-being of patients with migraine headache. Headache 34:337-343
3. Dahlöf CGH, Dimenäs E (1995) Migraine patients experience poorer subjective well-being/quality of life even between attacks. Cephalalgia $15: 31-36$ 
4. Bussone G, Usai S, Grazzi L, Rigamonti A, Solari A, D'Amico D (2004) Disability and quality of life in different primary headaches: results from Italian studies. Neurol Sci 25[Suppl 3]:S105-S107

5. Menken M, Munsat TL, Toole JF (2000) The Global Burden of Disease Study: implications for neurology. Arch Neurol 57:418-420

6. Schipper H (1983) Why measure quality of life? Can Med Assoc J 128:1367-1370

7. Carr AJ, Gibson B, Robinson PG (2001) Is quality of life determined by expectations or experience? BMJ 322:1240-1243

8. Patrick LD, Deyo RA (1989) Generic and disease-specific measures in assessing health status and quality of life. Med Care 27:S17-S31

9. Bayley BK, London MR, Grunkemeier GL, Lansky D (1995) Measuring the success of treatment in patient terms. Med Care 33:AS226-AS235

10. Santanello NC, Polis AB, Hartmaier SL, Kramer MS, Block GA, Silberstein SD (1997) Improvement in migrainespecific quality of life in a clinical trial of rizatriptan. Cephalalgia 17:867-872

11. Garber WH, Kosinski M, Dahlof C, Tepper S, Kujawski SC, Ware J, Batenhorst A (2001) HIT-6 reliably measures the impact of headache. Abstracts of the 10th. Congress of the International Headache Society. Cephalalgia 21:33
12. Diamond M, Neto W, Jacobs D, Fairclough D, Wolfe P, Papadopoulous G (2003) Quality of life measures from a large, randomized, double-blind, placebo-controlled study of topiramate in migraine prevention. Cephalalgia 23:710

13. D'Amico D, Santoro P, Frediani F, Bernardoni P, Misceo S et al (2003) Changes in disability and in quality of life scores in migraine patients after prophylaxis. Cephalalgia 23:745

14. Ciconelli RM (1997) Tradução para o português e validação do questionário genérico de avaliação de qualidade de vida "Medical Outcomes Study 36item Short-Form Health Survey (SF36)". Tese de doutorado. Universidade Federal de São Paulo

15. Ramadan NM, Silberstein SD, Freitag FG, Gilbert TT, Frishberg BM (2000) Evidenced-based guidelines for migraine headache in the primary care setting: pharmacological management for prevention of migraine. http://www.neurology.org

16. Adelman JU, Brod A, Von Seggern RL, Mannix LK, Rapoport AM (1998) Migraine preventive medications: a reappraisal. Cephalalgia 18:605-611

17. Tfelt-Hansen P (1995) Prophylactic treatment of migraine: evaluation of clinical trials and choice among drugs. Cephalalgia 15:29-32

18. Tfelt-Hansen P, Welch KMA (1993) Prioritizing prophylactic treatment. In: Olesen J, Tfelt-Hansen P, Welch KMA (eds) The headaches. Raven Press, New York, pp 403-407

19. Pascual J, Leira1 R, Láinez JM (2003) Combined therapy for migraine prevention? Clinical experience with a bblocker plus sodium valproate in 52 resistant migraine patients. Cephalalgia 23:961-962
20. Sandor OS, Afra J, Ambrosini A, Schoenen J (2000) Prophylactic treatment of migraine with beta-blockers and riboflavin: differential effects on the intensity dependence of auditory evoked cortical potentials. Headache 40:30-35

21. International Headache Society (2000) Guidelines for controlled trials of drugs in migraine. Members Handbook, pp 111-133

22. Dahlöf CGH (1995) Health-related quality of life under six months' treatment of migraine - an open clinicbased longitudinal study. Cephalalgia 15:414-422

23. Stewart AL, Hays RD, Ware JE (1988) The MOS Short-Form General Health Survey: reliability and validity in a patient population. Med Care 26:724-735

24. Brazier JE, Harper R, Jones NMB, O Cathian A, Thomas KJ, Unsherwood T, Westlake L (1992) Validating the SF-36 health survey questionnaire: new outcome measure for primary care. BMJ 305:160-164

25. Solomon GD, Skobieranda FG, Genzen JR (1995) Quality of life assessment among migraine patients treated with sumatriptan. Headache 35:449-454

26. Mushet GR, Miller DW, Clements B, Pait G, Gutteramn DL (1996) Impact of sumatriptan on workplace productivity, nonwork activities, and health-related quality of life among hospital employees with migraine. Headache 36:137-143

27. Salvesen R, Bekkelund SI (2003) Aspects of referral care for headache associated with improvement. Headache 43:779-783 